\title{
Use of microporous hydroxyapatite material in regenerative treatment of periodontal tissues in dogs: a clinical study
}

\author{
IZABELA POLKOWSKA, ANNA ŚLÓSARCZYK*, ALEKSANDRA SOBCZYŃSKA-RAK, \\ MAGDALENA GOŁYŃSKA, TOMASZ SZPONDER, BEATA ŻYLIŃSKA
}

\author{
Department of Veterinary Surgery, Faculty of Veterinary Medicine, \\ University of Life Sciences in Lublin, Głęboka 30 Street, Lublin, Poland \\ *Faculty of Materials Science and Ceramics, AGH University of Science and Technology, \\ Mickiewicza 30 Street, Kraków, Poland
}

Polkowska I., Ślósarczyk A., Sobczyńska-Rak A., Gołyńska M., Szponder T., Żylińska B. Use of microporous hydroxyapatite material in regenerative treatment of periodontal tissues in dogs: a clinical study

Summary

One of the biomaterials used in veterinary dentistry is hydroxyapatite (HAp). It aids the biological process of bone reconstruction and provides the basis on which damaged tissues can be rebuilt. It is also exceptionally osteoconductive and bioactive towards bone and other tissues. The aim of the present study was to verify the usefulness of hyd roxyapatite microporus ceramics for the treatment of periodontal diseases and post-extraction defects. The study was performed on 40 dogs. Dogs were qualified for the in vivo test: 2 study groups and 2 control groups $(\mathrm{K} 1, \mathrm{~K} 2)$ were created. Group G1 comprised 10 dogs diagnosed with periodontitis with 4-8 mm gingival pockets and mobility of mandibular/maxillary incisors. In order to avoid extraction, hydroxyapatite implantation into the bone pockets was performed. Group G2 comprised 10 dogs that required the extraction of maxillary canines, following which biomaterial was introduced into the post-extraction cavities. Control groups were performed without using of microporous hydroxyapatite. In group G1, animals displayed significant shallowing of gingival pockets. The mean depth of pockets was significantly reduced in those dogs and considerably better reconstruction of periodontal tissues was observed when compared to the control group K1. In group G2, significantly faster healing of bone cavities was stated when compared to the respective control group K2. The study confirmed the validity of using microporous hydroxyapatite granules and shaped blocks in reconstructive periodontal treatment as well as prevention of oronasal fistulas after canines extraction and facilitation of the post-extraction healing process.

Keywords: dog, microporous hydroxyapatite bioceramics, periodontal tissue, tissue regeneration

Periodontal diseases are among the most common oral lesions observed in carnivorous animals. According to literature, they affect over $80 \%$ of dogs aged three years or older (16). Untreated gingivitis may, relatively quickly, lead to periodontitis and impairment of the restorative processes of periodontal tissues. Metabolites produced by bacteria penetrate tissue inducing inflammation. Their cytotoxicity towards periodontal tissue results from enzymatic damage, they also suppress the organism's immunological mechanisms. The presence of inflammation causes damage to periodontal ligaments, bone resorption in the alveolar process on all interdental surfaces, and root furcations. It is harmful to epithelial attachment, gingival connective tissue, periodontium and root cementum $(6,7)$.
The goal of conventional periodontal treatment is to eliminate etiological factors and damaged tissue, as well as to stop the disease process. The subsequent therapeutic step is aimed at regenerating the damaged periodontal tissues and restoring its proper function. The reconstruction of damaged periodontal structures is done with the use of biomaterials (13). A number of regenerative techniques have been developed, including guided tissue regeneration (GTR) and guided bone regeneration (GBR). Considerable advances made in recent years in terms of the uses of implant materials in the treatment of periodontal diseases have greatly increased our ability to regenerate damaged tissues (19).

Rapid development in the use of various types of implant materials in periodontal reconstruction in 
humans has been observed in the $20^{\text {th }}$ century. In the 1950s, alloplastic materials were developed, which proved to be systematically well tolerated and functionally biocompatible with surrounding tissues (24). In the context of veterinary dentistry, various types of hydroxyapatite (HAp) based bone substitutes can be used. Hydroxyapatite may be derived from natural inorganic sources or synthesized. At present, mainly synthetic hydroxyapatite is widely used as bone substitute material. A large number of research activities has been dealing with the fabrication and application of hydroxyapatite ceramics. This hydrated calcium phosphate is described by the formula $\mathrm{Ca}_{10}\left(\mathrm{PO}_{4}\right)_{6}(\mathrm{OH})_{2}$ and constitutes the substance of enamel and dentine. As a bioactive material, after implantation, it binds with bone tissue and facilitates biological processes of bone reconstruction $(17,23)$. Moreover, it forms a frame for the regeneration of missing tissue, reduces the depth of gingival pockets and aids the regeneration of the connective tissue attachment (18). It induces the production of growth factors and populations of osteogenic cells (2). Numerous studies report that an important characteristic of biomaterial is the preservation of proper parameters in terms of porosity and granulation. In vivo research has revealed that porosity, pore size and geometry of hydroxyapatite implants affects both mechanical properties and biological behaviour after implantation. This is particularly important for tissue ingrowth (15). A rough implant surface provides osteoconductive qualities and allows for the retention of fibrin fibres, consequently aiding the formation of new bone directly in the implant's surface. The biomaterial's granulation is also important. Particles larger than 100 micrometers provide a space large enough for newly forming blood vessels and bone cells, facilitating accelerated in-healing of the biomaterial (21).

Bone disease processes such as inflammation, injury, cancer or post-surgery damage, may lead to bone loss and bone structure atrophy. Biomaterials applied at bone loss sites reduce the size of hematoma and have a beneficial effect on the healing process (22).

Regeneration of periodontal tissue is an increasingly important aspect of dentistry, human and veterinary alike. The use of bone graft materials allows the regeneration of tissues damaged in the course of a periodontal disease, as well as any other disease processes observed in the stomatognathic system, and the restoration of their proper function. Overall, full regeneration of periodontal tissue is uncommon due to, among other factors, the presence of pathogenic bacteria colonising the oral cavity and hindering regenerative processes. Moreover, tooth mobility may result in the destabilisation of the periodontal healing process. Therefore, the selection of a suitable material is a vital stage of any tissue engineering process (5).

The aim of the present study was to verify the validity of using microporous hydroxyapatite granules and shaped blocks in the treatment of periodontal diseases and bone loss resulting from mandibular fang extraction in dogs. For this reason, apart from using biomaterial in the treatment of post-extraction cavities, the study included an attempt to apply microporous hydroxyapatite ceramics in the treatment of periodontal diseases in dogs.

\section{Material and methods}

In our in vivo studies microporous hydroxyapatite material of controlled homogenous pore size distribution and pore sizes within the range of a hundredth and tenth parts of a micrometer were applied. The above material was developed at the Faculty of Materials Science and Ceramics, University of Science and Metallurgy, Kraków, Poland. The initial hydroxyapatite powder was obtained by the wet chemical method. The granules and shaped blocks were obtained directly from the filter cake after its drying followed by calcination at $800^{\circ} \mathrm{C}$. Fabricated material shows a high water absorption ability, which is a particularly advantageous feature in medical applications. In animal experiments microporous HAp granules $(0.3-0.5 \mathrm{~mm})$ and shaped blocks with $\sim 68$ vol.\% porosity and average pore size $0.1 \mu \mathrm{m}$ were applied.

The study was conducted on 40 dogs treated at the Department and Clinic of Veterinary Surgery at the University of Life Sciences in Lublin, divided into 2 groups. The first group comprised 2 sub-groups: a study group (G1) and control group (K1) with 10 dogs each. Similarly, the second group comprised a study (G2) and control (K2) sub-groups. Dogs qualified for the study were selected for systemic diseases based on medical history, clinical examination and the examination of blood parameters. Dental examinations of patients were performed under general anaesthesia. Prior to the collection, the owners of dogs qualified for the study and were informed of the purpose of the present study and gave approval for their participation.

The dominant clinical symptoms in all animals from the first group (G1, K1) included: feeding disorders and fetor ex ore. The following were evaluated: gingival index (GI), depth of periodontal pockets (PPD), clinical attachment level (CAL) and tooth mobility (TM) (Tab. 1). Based on the above parameters, the severity of periodontal inflammation was assessed (the fourth stage of PD). Prior to therapy, radiological examinations were also performed in order to determine the extent of damage to the alveolar process.

The gingival index (GI) was established after Löe and Solness. It was rated on the following scale: 0 - healthy gingiva (gum light pink in colour), 1 - mild inflammation - minor colour changes and slight swelling, no bleeding during probing, 2 - moderate inflammation - reddening, swelling and gingival gloss, bleeding present during probing, 3 - severe inflammation - strong reddening, swelling, ulceration and incidence of spontaneous gingival bleeding.

The examination of pocket depth using a periodontal probe and assessment of connective tissue attachment (the distance from the cemento-enamel-junction to the bottom of the pocket) was performed with the use of a Williams periodontal probe. Tests were performed in four locations at the surface of all incisors (mesial and distal labial and lingual side). The sum of all obtained values was divided by four to obtain the index value for the particular tooth. Subsequently, values obtained for individual teeth were added up and divided by the number of teeth examined. Patients qualified for the study were diagnosed with mandibular or maxillary incisor gingival 
pockets of $\geq 4 \mathrm{~mm}$ and loss of connective tissue attachment of $\geq 1 \mathrm{~mm}$. On the basis of the above, the animals were classified under one of the following three categories depending on their PPD index: 1-3 mm - physiological depth of the gingival sulcus, 3-4 mm - mild periodontitis, 4-6 mm - moderate periodontitis, 6-9 $\mathrm{mm}$ - severe periodontitis.

The value (in $\mathrm{mm}$ ) obtained from the measurement of connective tissue attachment level was used as a reference value in the assessment of ongoing treatment.

Tooth mobility was measured with a probe. 4 classes of mobility were adopted: 0 - no mobility, 1 - lateral tooth mobility of $1 \mathrm{~mm}$ or less, 2 - lateral tooth mobility of over $1 \mathrm{~mm}, 3$ - lateral and vertical tooth mobility.

All indexes were measured at the onset of treatment and then during subsequent checkups. The result of dental examinations are presented in Table 1.

Groups G1 and K1 were determined by dogs diagnosed, on the basis of the GI index, with moderate or severe periodontitis, considerable inflammation of periodontal tissues, presence of 4-9 $\mathrm{mm}$ gingival pockets and significant loss of connective tissue attachment. Due to gingival recession and alveolar bone atrophy in the course of periodontitis, the patients also suffered from maxillary or mandibular incisors mobility (mobility class 2 and 3). All animals underwent dental treatment. The therapy included removal of tartar from tooth crowns (supragingival tartar), gingival pockets and root surfaces (subgingival tartar), followed by polishing tooth crowns. In ten dogs $(\mathrm{G} 1)$, in order to avoid extraction

Tab. 1. The results of the dental examination in research groups

\begin{tabular}{|c|c|c|c|c|}
\hline \multirow[b]{2}{*}{ Breed } & \multicolumn{2}{|c|}{ Before treatment } & \multicolumn{2}{|c|}{ After treatment } \\
\hline & GI & $\begin{array}{l}\text { PPD mean } \\
(\mathrm{mm})\end{array}$ & GI & $\begin{array}{l}\text { PPD mean } \\
(\mathrm{mm})\end{array}$ \\
\hline \multicolumn{5}{|c|}{ Group G1 } \\
\hline Mixed breed, 3 , age 5 & 2.0 & 7.2 & 0.5 & 4.3 \\
\hline Pinsher, $\widehat{O}$, age 2 & 2.0 & 5.5 & 0.87 & 4.0 \\
\hline Terier, $\widehat{\jmath}$, age 3 & 1.5 & 5.3 & 0.25 & 3.7 \\
\hline 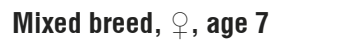 & 2.5 & 8.0 & 0.75 & 6.2 \\
\hline Mixed breed, ${ }^{\lambda}$, age 9 & 1.75 & 6.6 & 0.25 & 4.1 \\
\hline German Shepherd, ô, age 3 & 2.25 & 6.3 & 0.58 & 4.4 \\
\hline German Shepherd, ô, age 3 & 1.5 & 4.0 & 0.25 & 2.5 \\
\hline Terrier, $q$, age 5 & 2.0 & 7.0 & 0.25 & 5.2 \\
\hline Cocer spaniel, $q$, age 2 & 1.5 & 5.3 & 0.25 & 4.5 \\
\hline Mixed breed, 3 , age 3 & 2.0 & 6.8 & 0.25 & 3.7 \\
\hline \multicolumn{5}{|c|}{ Group K1 } \\
\hline Mixed breed, $\widehat{C}$, age 5 & 1.5 & 4.2 & 1.12 & 3.8 \\
\hline Mixed breed, $\circ$, age 2 & 1.75 & 7.1 & 1.5 & 6.6 \\
\hline Dachshund, $\widehat{\jmath}$, age 5 & 1.75 & 5.4 & 1.62 & 5.2 \\
\hline Mixed breed,, , age 4 & 1.12 & 4.8 & 0.91 & 4.0 \\
\hline Mixed breed, 3 , age 2 & 2.0 & 6.7 & 1.5 & 6.2 \\
\hline German Shepherd, $q$, age 3 & 1.25 & 4.8 & 0.91 & 4.5 \\
\hline 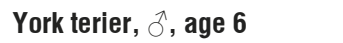 & 1.66 & 5.6 & 1.12 & 4.9 \\
\hline Mixed breed, $\circ$, age 4 & 1.5 & 6.4 & 1.12 & 5.8 \\
\hline Mixed breed, $\widehat{\partial}$, age 3 & 2.0 & 5.5 & 1.5 & 5.0 \\
\hline German Shepherd, ${ }^{1}$, age 3 & 2.0 & 6.0 & 1.66 & 5.6 \\
\hline
\end{tabular}

of incisors, hydroxyapatite implantation into bone pockets was performed. The HAp was applied in the form of 150$-500 \mu \mathrm{g}$ granules saturated with gentamicinin (Fig. 3, 4). The implantation was secured with absorbable yarn. Periodontal tissue checkups were performed 8 weeks after the procedure. The control group K1 was determined by dogs, in which only conventional treatment was performed, without using hydroxyapatite ceramics.

Groups G2 and K2 were determined by dogs in which, due to significant tooth mobility apical lesions or maxillary bone damage and atrophy, canine extractions were required. Among clinical symptoms in these dogs were sneezing and unilateral mucopurulent nasal discharge. In all of these dogs necessary extractions were performed. In group G2 each post-extraction cavity was implanted with biomaterial in the form of blocks sized to match particular cavities (Fig. 5). Mucosa was stitched together with absorbable yarn. Check-up examinations were performed 4 months after implantation. The control group K2 was a reflection of the study group (G2) without using hydroxyapatite shaped blocks implanted into extraction pockets.

Depending on the severity of inflammatory changes in periodontal tissues in all of patients weekly antibiotic therapy was used.

Statistical analysis. A statistical analysis was conducted to establish whether any significant correlations could be observed between the gingival pocket depth index and the incidence of gingivitis prior to treatment and after the therapy, both in the study group and the control group. To verify normal distribution of the numeric data, the Shapiro-Wilk test was used. For the comparison between the particular groups, the t-Student test was used.

\section{Results and discussion}

Eight weeks after dental treatment and the introduction of hydroxyapatite granules into gingival pockets, Group 1 animals displayed significant shallowing of gingival pockets. The mean depth of pockets was significantly reduced in all animals in this group (by 3-4 mm) when compared to the original measurement (T-Student level of $p<0.0001)$. The results of the statistical analysis are presented in Fig. 1. Mean values of gingival index (GI) and clinical attachment level (CAL) were significantly reduced $(p<0.0001)$. Considerable regeneration of connective tissue attachment and improved tooth stability was observed in clinical study which allowed the avoidance of tooth extraction (Tab. 1).

In Group 2, following canines extraction and implantation with hydroxyapatite blocks, a considerable acceleration of bone cavity healing was observed. Dental imaging performed after 4 months from biomaterial implantation revealed that $50 \%$ of the implant had been reconstructed and replaced with bone tissue (Fig. 6). Full osteointegration was observed after 8-12 months from the treatment.

In control group $\mathrm{K} 1$, after 8 weeks a slight reduction of periodontal pocket depth was observed, and in these weeks after the dental treatment, patients started coming back to the Clinic due to a relapse of periondotitis, which in most cases necessitated extraction of unstable incisors. 


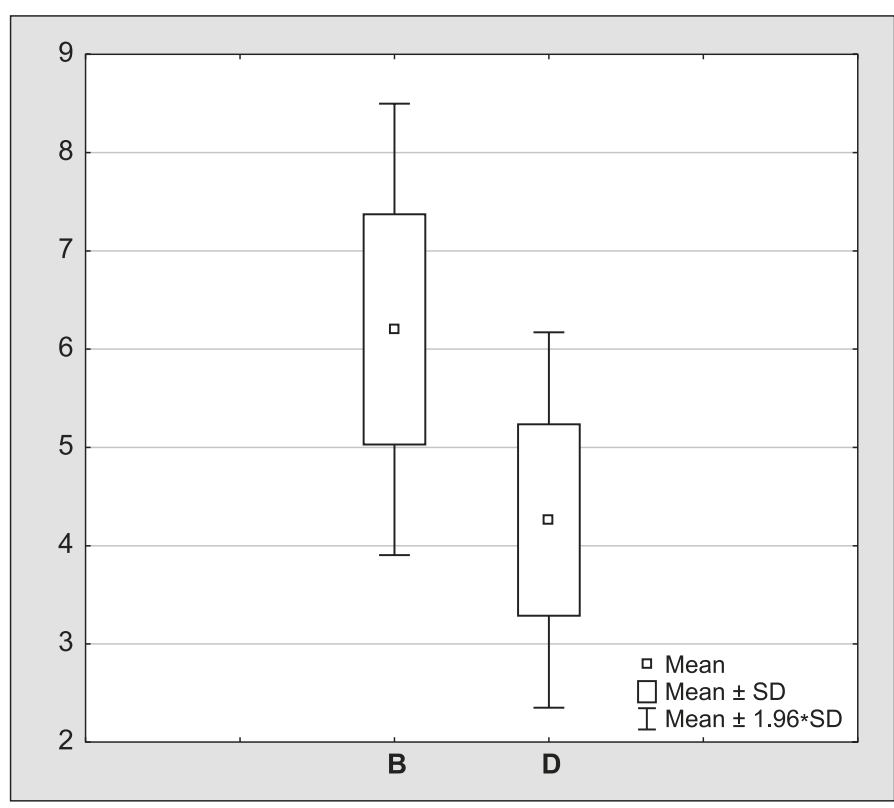

Fig. 1. Comparison of the gingival pocket depth index in the study group (Group G1) before and after hydroxyapatite treatment

Explanations: B - Group G1: gingival pocket depth index (PPD) before treatment; D - Group G1: gingival pocket depth index (PPD) after hydroxyapatite treatment

In the analysis of the PPD index after hydroxyapatite treatment in the study group (G1), as compared to the $\mathrm{K} 1$ control group results without the use of biomaterial, significantly lower means were observed in the study group (Fig. 2).

Statistical data confirm the viability of the study as the observed periodontal pocket depth was significantly reduced after hydroxyapatite treatment (Group I), as compared to the values reported in the control group (K1) - Tab. 2.

In control group $\mathrm{K} 2$, where maxillary canines extraction had been necessary, the lack of biomaterial implantation lead to complications in the form of oronasal fistulas, which developed in $60 \%$ of the cases and required further intensive treatment. In the remaining $20 \%$ of the cases, the bone healed correctly, however, dental imagery revealed incomplete filling of the postextraction cavities with bone tissue.

The purpose of performing reconstructive procedures with the use of biomaterials is in human and veterinary medicine alike to recreate the integrity of damaged tissue and restore its proper function. Reconstructive materials most commonly used in dentistry include autogenic bone grafts and alloplastic materials (8). Among the latter, hydroxyapatite, used in human as well as veterinary

Tab. 2. Comparison of the periodontal pocket depth index in the study group (Group G1) after hydroxyapatite treatment and the control group (K1) after treatment without the use of hydroxyapatite

\begin{tabular}{|c|c|c|c|c|c|}
\hline Group & Mean & SD & $\mathrm{n}$ & $\mathrm{t}$ & $p$-Value \\
\hline G1 & 4.260000 & 0.974337 & 10 & 2.13267 & 0.046976 \\
K1 & 5.160000 & 0.911897 & & & \\
\hline
\end{tabular}

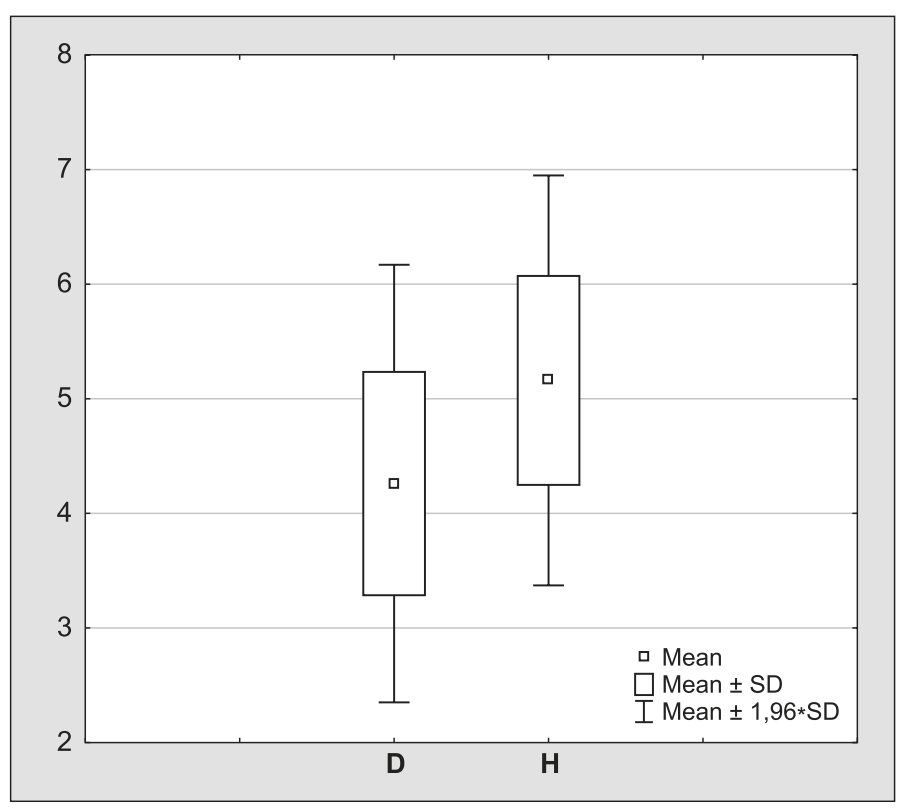

Fig. 2. Comparison of the gingival pocket depth index in the study group (Group G1) after hydroxyapatite treatment and the control group (K1) after treatment without the use of biomaterial

Explanations: D - Group G1: gingival pocket depth index (PPD) after hydroxyapatite treatment; H - Group K1: gingival pocket depth index (PPD) after treatment without the use of microporous hydroxyapatite ceramics

dentistry, has become a model compound reflecting the bone mineral phase. It is considered to be a leading biomaterial in bone surgery due to its high biocompatibility, osteocondactivity and osteoinductivity as well as capacity to form chemical bonds with living tissue (9, 12). Dense hydroxyapatite materials are not resorbable and bone reconstruction takes place around the material particles (22). In porous forms, they undergo biodegradation and are gradually replaced with regenerating bone. In dental surgery, hydroxyapatite is typically used in the form of lamellas, granules or blocks of various shapes and sizes. The size of HAp granules and the diameter of pores in the range of macro and microporosity impact the effects of bone reconstruction. The smaller the granules, the greater the area of interaction formed and the faster the material's resorption (Fig. 2, 3). The minimum size of open pores allowing for the formation of a biological bond between the implant and the bone is $100 \mu \mathrm{m}(10)$. Porosity of the implant material also facilitates the conduction of osteoblasts, oxygen perfusion, and angiogenesis in the newly formed bone tissue. If not enough space is left, bone tissue growth becomes impossible (25). Hydroxyapatite induces the production of growth factors and populations of osteogenic cells, and by ensuring a favourable chemical environment, it facilitates the formation of new bone (11).

In human dentistry it is common to use biomaterials in the treatment of bone defects, a technique known as guided bone regeneration (GBR). Hydroxyapatite used as a filling for bone cavities forms a matrix for the production of new bone tissue. It has been demonstrated to be non-immunogenic and non-toxic. After implantation, 


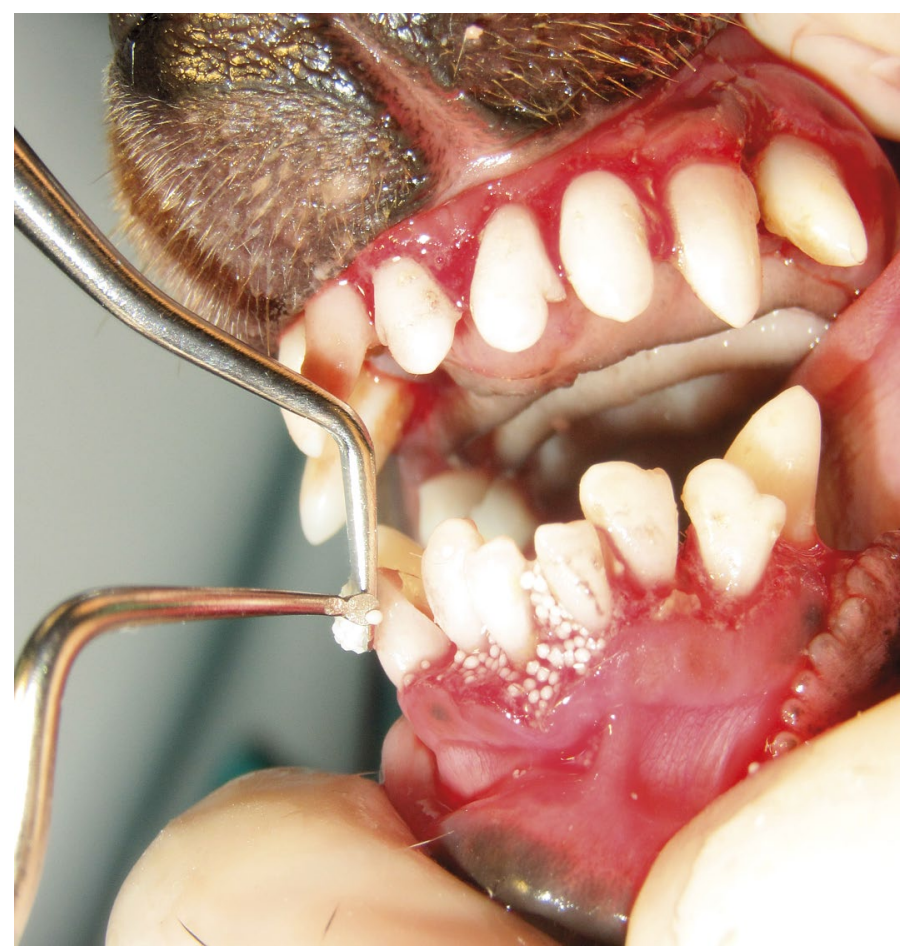

Fig. 3. Application of microporous HAp granules in a gingival pocket

a porous structure is formed in place of the bone cavity, which facilitates the process of tissue regeneration and limits gingival recession (3). Due to its osteoconductivity, it provides a perfect base for osteogenic cell adhesion, which helps the formation of new bone $(7,18)$. HAp stimulates more active tissue regeneration when compared to a purely natural process (5).

In veterinary medicine, bone reconstruction with the use of biomaterials remains under clinical investigation. Song et al. (20) demonstrated in their research a higher resistance to femoral bone damage in dogs implanted with a prosthesis in the form of a synthetic femoral bone coated with hydroxyapatite versus autogenic bone grafts introduced into the medullary cavity. Other researchers also confirmed that a hydroxyapatite coated prosthesis may improve the efficiency of bone tissue growth and ensure a good connection with the natural bone (1).

In their study of hydroxyapatite, Lee et al. (14) demonstrated its effectiveness as a substitute for an autogenic shinbone graft in rabbits. It was used in the form of blocks and powder. The researchers concluded that hydroxyapatite facilitated consolidation with the host bone tissue.

In the context of veterinary dentistry, a commonly observed type of bone damage (vertical) occurs along the root and towards the crown of the tooth, which is the reason why oronasal fistulas are so common in dogs (most often located in the vicinity of the canines on the palatal side). Fistulas are also formed as a result of periapical lesions or extraction of maxillary canines. The healing of a post-extraction cavity is not dissimilar to the processes of new tissue formation observed in instances of long bone fractures (4). Tissue regeneration affects the bone edges of the wound as well as the inner surfaces

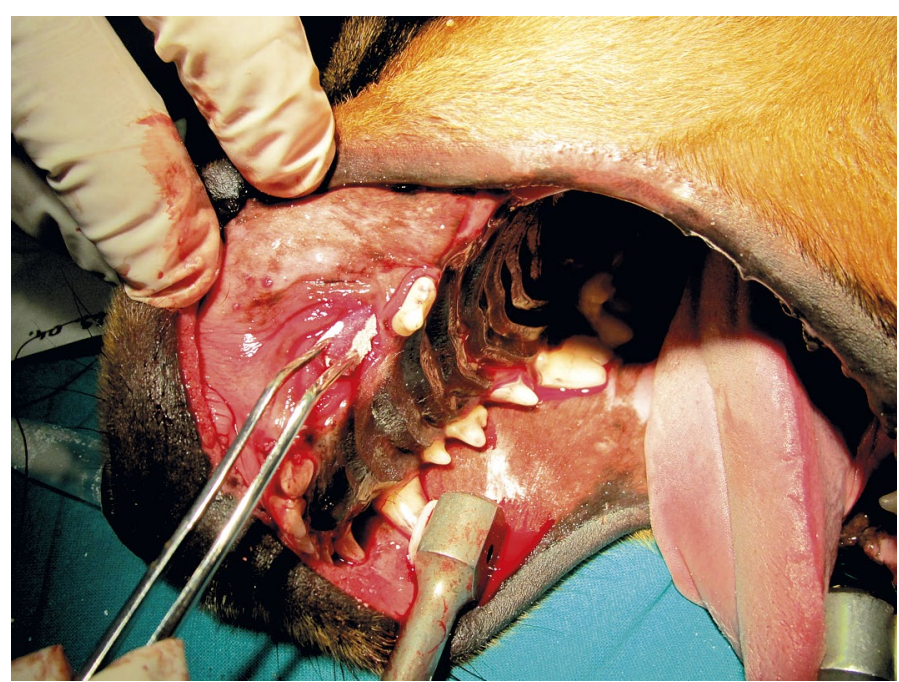

Fig. 4. Implantation of a microporous HAp block shaped to fit the post-extraction cavity

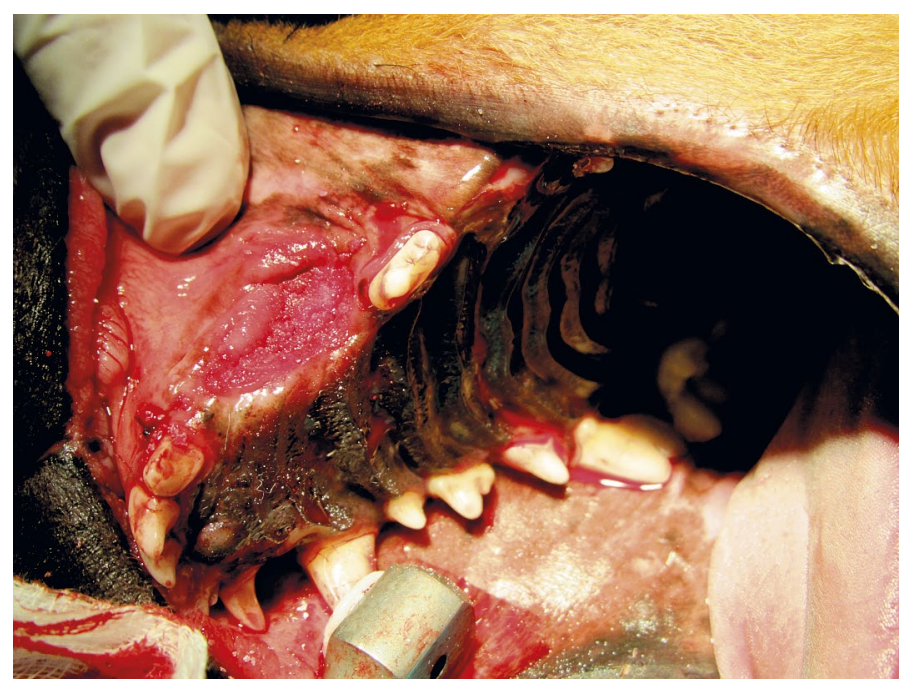

Fig. 5. Interstitial fluids permeability at the implantation site

of alveolar walls. It involves a combination of intra and extra alveolar processes of reformation and resorption of bone tissue. Connective tissue proliferates into the alveolus, in effect reducing the height of the alveolar process edge. It is relatively rare for the cavity to be completely filled with properly formed bone tissue (18). The use

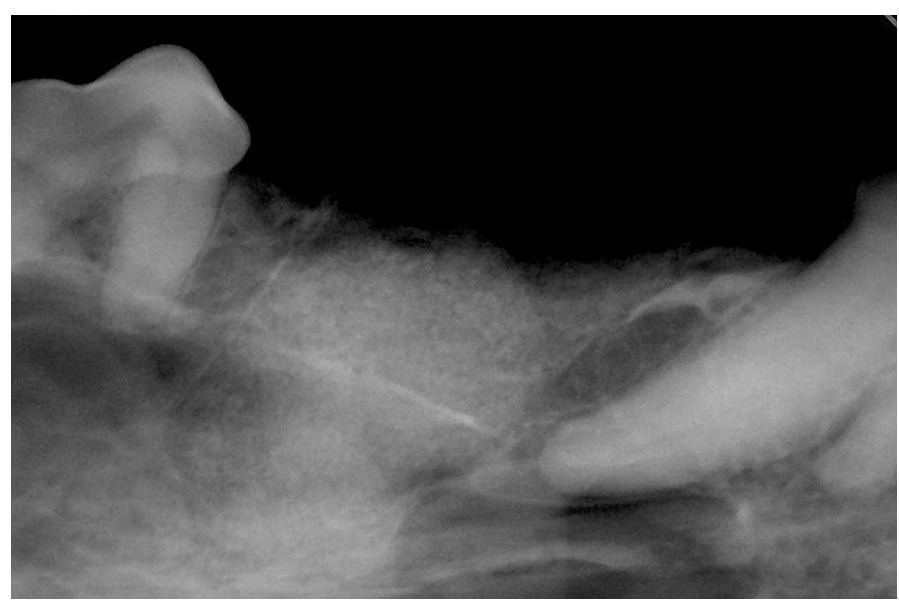

Fig. 6. Biomaterial healing: X-Ray evaluation 2 months after the procedure 
of biomaterials offers a solution to this problem. Under optimum implantation conditions in the area of cell embedding, the cells' ability to reorganise and form new tissue is greatly facilitated. In our study, complications of this type were observed in control group K2 animals following maxillary canines extraction, where in some cases the post-extraction cavity was still not fully filled with bone after 1 year from the procedure. Meanwhile in Group 2 animals, where microporous hydroxyapatite bioceramics was used in combination with antibiotics, proper implant in-healing was observed and the fistulas closed within as little as 2 weeks. Complete filling of the post-extraction cavity with properly structured bone tissue was observed within 8-12 months from the procedure.

The clinical potential of bone substitute materials allows for their widespread use in various areas of human dentistry. Apart from treatment of bone damage, hydroxyapatite has become a common material used in periodontal regeneration related to the presence of bone pockets and tooth furcactions. Its application allows the improvement of tooth stability and radiologically confirmed reduction of bone pocket depth (22).

In one of the study groups (Group 1), the implantation of microporous hydroxyapatite granules soaked with gentamicin into gingival pockets significantly accelerated the healing of periodontal tissues and reduction of gingival pockets. Local administration of the antibiotic allowed for a focused therapeutic concentration. Satisfactory results of the treatment could be observed as early as eight weeks after the procedure. The restorative qualities of hydroxyapatite facilitated significant shallowing of gingival pockets, healing of the implantation areas, and stabilising mobile teeth. No such results were observed in the control group (K1) subjected to scaling combined with general antibiotic treatment. In this group, the gingival pockets were only slightly shallower and many patients returned to the clinic after a short period of time with symptoms of periodontitis.

In clinical cases of oral inflammation in animals, an important therapeutic objective is to ensure regeneration of the periodontium and bone with the possible use of biomaterials, including microporous hydroxyapatite bioceramics. In dogs with periodontopathy, the use of above hydroxyapatite accelerates the healing process and saturated with gentamycin limits the development of inflammation. Clinical evaluation confirms that hydroxyapatite enhances regeneration of tooth support tissues and restoration of their proper function. In dogs, it has also been used as a filling for post-extraction cavities. It speeds up the reconstruction of bone tissue. The performed study confirms the validity of using microporous granules and shaped hydroxyapatite blocks in veterinary dentistry as filling for facio-maxillary bone defects and bone pockets. The economical aspect of the same is also noteworthy. The cost of the biomaterial is significantly lower than that of other available implantation materials, which further adds to its attractiveness in the context of veterinary medicine.

\section{References}

1. Al Hertani W., Waddell J. P., Anderson G. I.: The effect of partial vs. full hydroxyapatite coating on periprosthetic bone quality around the canine madreporic femoral stem. J. Biomed. Mat. Res. 2000, 53, 518-524.

2. Bansal S., Chauhan V., Sharma S., Maheshwari M., Juyal A., Raghuvanshi S.: Evaluation of hydroxyapatite and beta-tricalcium phosphate mixed with bone marrow aspirate as a bone graft substitute for posterolateral spinal fusion. Indian J. Orthop. 2009, 43, 234-239.

3. Belcarz A., Ginalska G., Zima A., Polkowska I., Ślósarczyk A., Szyszkowska A.: New HAp-organic composite as a promising filler of bone defects. Engin. Biomat. 2009, 12, 11-15.

4. Cardaropoli G., Araújo M., Lindhe J.: Dynamics of bone tissue formation in tooth extraction sites. An experimental study in dogs. J. Clin. Periodontol. 2003, 30, 809-818.

5. De Santis E., Botticelli D., Pantani F., Pereira F. P., Beolchini M., Lang N. P.: Bone regeneration at implants placed into extraction sockets of maxillary incisors in dogs. Clin. Oral. Implants. Res. 2011, 22, 430-437.

6. Doi K., Kubo T., Takeshita R., Kajihara S., Kato S., Kawazoe Y., Shiba T., Akagawa $Y$.: Inorganic polyphosphate adsorbed onto hydroxyapatite for guided bone regeneration: an animal study. Dent. Mater. J. 2014, 33, 179-186.

7. Doi K., Oue H., Morita K., Kajihara S., Kubo T., Koretake K., Perrotti V., Iezzi G., Piattelli A., Akagawa Y.: Development of implant/interconnected porous hydroxyapatite complex as new concept graft material. Plos One 2012, 7, 1-10.

8. Dominiak M., Eysiak K.: Evaluation of effectiveness of selected guide bone regeneration method with usage biomaterial in the treatment of the defects of alveolar process after teeth extractions - Preliminary study. Dent. Med. Probl. 2006, 43, 368-378.

9. Hong J. Y., Lee J. S., Pang E. K., Jung U. W., Choi S. H., Kim C. K.: Impact of different synthetic bone fillers on healing of extraction sockets: an experimental study in dogs. Clin. Oral Implants. Res. 2014, 25, 30-37.

10. Jelonek A., Skórska-Stania A., Myciński P., Zarzecka J.: Hydroxyapatite microspheres coated with poly (D, L- lactide) as bone filler and antibiotic delivery system. Engin. Biomat. 2013, 16, 19-29.

11. Jung U. W., Hwang J. W., Choi D. Y., Hu K. S., Kwon M. K., Choi S. H., Kim $H$. J.: Surface characteristics of a novel hydroxyapatite-coated dental implant. J. Periodontal Implant Sci. 2012, 42, 59-63.

12. Jung U. W., Kim S., Lee I. K., Kim M. S., Lee J. S., Kim H. J.: Secondary stability of microthickness hydroxyapatite-coated dental implants installed without primary stability in dogs. Clin. Oral Implants Res. 2013, 25, 1169-1174.

13. Lee J. S., Park W. Y., Cha J. K., Jung U. W., Kim C. S., Lee Y. K., Choi S. H.: Periodontal tissue reaction to customized nano-hydroxyapatite block scaffold in one-wall intrabony defect: a histologic study in dogs. J. Periodontal Implant Sci. 2012, 42, 50-58.

14. Lee M. J., Sohn S. K., Kim K. T., Kim C. H., Ahn H. B., Rho M. S., Jeong M. H., Sun $S . K$.: Effect of hydroxyapatite on bone integration in a rabbit tibial defect model. Clin. Orthop. Surg. 2010, 2, 90-97.

15. Li S. H., De Groot K., Layrolle P.: Bioceramic scaffold with controlled porous structure for bone tissue engineering. Key. Eng. Mater. 2002, 218-220, 25-30.

16. Pieri F. A., Falci Daibert A. P., Bourguignon E., Scatamburlo Mortira M. A.: A Bird's-Eye View of Veterinary Medicine Source. Chapter: Periodontal disease in dogs. Ed. Carlos C. Perez-Marin. In Tech, Rijeka 2012, p. 119-140.

17. Polkowska I., Sobczyńska-RakA., Szyszkowska A., Koper J., Ślósarczyk A.: Use of hydroxyapatite in the treatment of bone cavities after the removal of inflammatory lesions within the jaws - case reports. Veter. Med. 2012, 68, 436-440.

18. Shue L., Yufeng Z., Mony U.: Biomaterials for periodontal regeneration: a review of ceramics and polymers. Biomatter. 2012, 2, 271-277.

19. Sobczak A., Kowalski Z., Wzorek Z.: Preparation of hydroxyapatite from animal bones. Acta Bioeng. Biomech. 2009, 11, 23-28.

20. Song K. G., Ma D. H., Hou J. W., Wang H., Yan J. L., Yu Z. G., Liang Z. Z.: Effect of morselized bones on binding of the canine hydroxyapatite-coated femoral stem. Chin. Med. J. 2010, 123, 1163-1169.

21. Szyszkowska A., Koper J., Piskorz T., Szczerba J., Puławska M.: Materials supporting bone regeneration in oral surgery - literature review. Implantoprosthetics 2010, 38, 9-12.

22. Szyszkowska A., Krawczyk P.: Materials used to regeneration bones cavities in stomatology. A review article. Implantoprosthetics 2008, 4, 21-24.

23. Vahabi S., Amirizadeh N., Shokrgozar M. A., Mofeed R., Mashhadi A., Aghaloo M., Sharifi D., Jabbareh L. A.: comparison between the efficacy of Bio-Oss, hydroxyapatite tricalcium phosphate and combination of mesenchymal stem cells in inducing bone regeneration. Chang Gung Med. J. 2012, 35, 28-37.

24. Wierucka-Mtynarczyk B., Hüpsch-Marzec H., Kubicka-Musiat M.: Advantages and limitations of using graft materials in periodontal treatment with particular regard to silated hydroxyethylcellulose. Dent. Med. Probl. 2010, 47, 365-372.

25. Yang C., Lee J. S., Jung U. W., Seo Y. K., ParkJ. K., Choi S. H.: Periodontal regeneration with nano-hyroxyapatite-coated silk scaffolds in dogs. J. Periodontal. Implant Sci. 2013, 43, 315-322.

Corresponding author: Izabela Polkowska, Associate Professor UP, ul. Glęboka 30, 20-612 Lublin, Poland; e-mail: iza.polkowska58@gmail.com 